\title{
SITES SOBRE DROGAS DE ABUSO: RECURSOS PARA AVALIAÇÃO
}

SITES ON DRUGS OF ABUSE: RESOURCES FOR ASSESSMENT

\author{
Vagner Santos ${ }^{1}$ \\ Marcelle Maria Portal ${ }^{2}$ \\ Maristela Ferigolo ${ }^{3}$ \\ Denise Conceição Mesquita Dantas ${ }^{4}$ \\ Helena Maria Tannhauser Barros ${ }^{5}$ \\ Carolina Sturm Trindade ${ }^{6}$
}

Resumo Os profissionais da saúde devem ser estimulados a adquirir independência própria na busca, seleção e utilização de informações para solução de problemas do dia-a-dia. Além disso, devem ser incentivados a refletir sobre os fatos vividos, visando à reconstrução da trajetória percorrida, dando-lhes um novo significado. Este texto apresenta uma experiência de aprendizagem, vivenciada por estudantes de graduação, durante o processo de avaliação de sites brasileiros sobre drogas de abuso. Mediante a identificação do grande número de informações sobre drogas de abuso e da falta de critérios para sua divulgação na internet, iniciou-se um processo de pesquisa que desencadeou a construção de um protocolo para auxiliar o processo de seleção de sites brasileiros que abordam o tema drogas de abuso.

Palavras-chave internet; informática em saúde; drogas de abuso.
Abstract Health professionals should be encouraged to gain independence in the search for, selection, and use of information to solve daily problems. Furthermore, they should be encouraged to reflect on the events that were experienced, to pursue the reconstruction of the path that was followed, and to give them a new meaning. This article presents a learning experience graduate students had during the process of evaluating Brazilian sites on drugs of abuse. After ascertaining the great amount of information on drugs of abuse and the lack of criteria in discussing them on the Internet, a research process got underway that triggered the preparation of a protocol to assist in the process of selecting Brazilian sites that address the drugs of abuse issue.

Keywords Internet; information technology in health, drugs of abuse. 


\section{Introdução}

O profissional de saúde deve ser preparado para buscar informações de forma crítica, incentivando-se a autoaprendizagem. No caso do uso de informações, isso significa adquirir independência própria na busca, seleção e utilização de informações para solução de problemas do dia-a-dia. O crescimento da internet é um dos maiores acontecimentos que está sendo vivenciado na atualidade, pois se converteu em um meio mais dinâmico e estimulante para encontrar e recuperar informações em saúde (Sanz-Valero et al., 2006). Ensinar a ler e a escrever - e também a pensar e a pesquisar - significa, hoje em dia em uma cultura digital, ensinar a utilizar os novos meios de informática e comunicação, despertando o interesse pelo desenvolvimento de si mesmo, como indivíduo e como comunidade, com vistas a uma cultura que se entrelaça no face to face e na interface (Glowniak e Bushway, 1994).

O presente trabalho apresenta um relato de experiência de aprendizagem resultante da vivência de estudantes de graduação na atividade de avaliação de sites brasileiros sobre drogas de abuso.

Estudos na área da educação têm mostrado que, por meio das novas experiências, ampliam-se novos conhecimentos e habilidades (Alheit e Dausien, 2006). Nesse sentido, tornam-se importantes os relatos de experiências, pois, quando uma pessoa relata os fatos vividos por ela mesma, percebe-se que reconstrói a trajetória percorrida dando-lhe um novo significado.

Essa narrativa demonstra a vivência prática que desencadeou o problema, o compromisso social investido na pesquisa, as hipóteses desenvolvidas, bem como a importante rede de amparo que esteve presente para o desenvolvimento de novas competências. Nessa experiência de aprendizagem, o conhecimento foi construído por descobertas sobre as diferentes possibilidades, concedendo-se liberdade para criar e construir (Freire, 2000). A importância dessa atividade é que se proporcionaram, além do desenvolvimento de habilidades e de competências referentes ao tema estudado, reflexões sobre como transcorreu o processo de aprendizagem.

\section{Uma visão global da realidade: drogas e internet}

O abuso de drogas tem-se destacado como importante problema de saúde pública no Brasil. Conforme o II Levantamento Domiciliar sobre o Uso de Drogas Psicotrópicas no Brasil, realizado em 2005, excetuando-se álcool e tabaco, 22,8\% da população pesquisada nas 108 maiores cidades brasileiras já fizeram uso de drogas na vida, o que corresponde a uma população de 10,7 milhões de pessoas (Carlini, 2007). Apesar da alta taxa de prevalência referente ao uso de drogas psicotrópicas e das múltiplas consequências adversas 
relacionadas ao seu consumo, poucos profissionais da saúde recebem treinamento para atendimento nessa área (Fleming e Murray, 1998).

Do reconhecimento de ambos os problemas, da alta prevalência e da falta de treinamento de profissionais para atendimento qualificado, surge o Serviço Nacional de Orientações e Informações sobre a Prevenção ao Uso Indevido de Drogas (VivaVoz). Trata-se de um serviço de atendimento telefônico gratuito, anônimo, que funciona como um call center, com a finalidade de prestar orientações e informações sobre a prevenção do uso indevido de drogas à população brasileira (Brasil, 2004). Esse atendimento é prestado por consultores, na sua maioria alunos de graduação. Esses estudantes recebem capacitação específica, direcionada para conceder corretamente esclarecimentos acerca das características de drogas psicoativas, suas ações no organismo, prevenção ao seu uso, bem como orientar sobre os recursos disponíveis na comunidade para quem precisa de algum tipo de atenção (Souza et al., 2006).

No Brasil, segundo levantamento realizado pelo Comitê Gestor de Internet, a proporção de indivíduos que utilizam a internet para busca de informações e serviços on line é de $88 \%$. Dentre esses, 39\% utilizam a internet para busca de informação relacionada à saúde ou serviços de saúde (Brasil, 2009). No entanto, não há noção exata sobre os riscos a que esses indivíduos estão expostos, quando essa informação estiver errada (Gunther, 2002a).

\section{Nosso contexto: informação de qualidade sobre drogas de abuso}

Infelizmente, reconhece-se que o conteúdo do material disponibilizado na internet pode ser de qualidade variável e de fonte duvidosa e, no caso das drogas, isso não é diferente. Logo, a busca por informação de qualidade na internet torna-se uma tarefa dispendiosa, independentemente de quem a faça: 'usuários leigos', profissionais da área da saúde, estudantes e, também, os consultores do VivaVoz.

Para o desenvolvimento do trabalho dos consultores, o VivaVoz disponibiliza diversos recursos como, por exemplo, software desenvolvido especificamente para registro dos atendimentos telefônicos, banco de dados atualizado sobre drogas de abuso e dependência química, biblioteca específica para o tema, supervisão contínua de profissionais formados e acesso irrestrito à internet.

O treinamento dos consultores no VivaVoz baseia-se em um modelo de ensino em neurociências sobre drogas de abuso, envolvendo aprendizagem sobre substâncias psicoativas e dependência química. O processo de capacitação engloba treinamento teórico (trinta horas) e, posteriormente, treinamento prático (vinte horas) (Barros, 2008). Esse modelo educacional representa uma 
adaptação do Medical Education for the Prevention and Treatment of Alcohol Use Disorders (Fleming e Murray, 1996; 1998). Por meio dos treinamentos, o consultor desenvolve aptidão para realizar busca de dados/informações em bases biomédicas reconhecidas. No entanto, alguns consultores não se sentiam aptos para avaliar adequadamente as informações disponíveis em sites comuns, local onde a maioria dos usuários leigos procura informação.

A partir dessa constatação gerou-se um questionamento: Quais sites poderiam constar na lista de favoritos dos computadores usados pelos consultores? A resposta para essa questão foi fator motivador da pesquisa. Inicialmente, a inclusão de sites na lista de favoritos baseou-se nas seguintes justificativas: 'são bons', 'o site é do governo', 'é um especialista que assina o site'. Entretanto, não se conseguia explicar, justificar ou conceituar, por exemplo, 'o que é bom'. Nesse momento, ficou claro que existia um problema maior: a falta de preparo frente às questões relativas à avaliação crítica das informações relacionadas às drogas, quando disponibilizadas nas páginas da internet.

Assim, tinha-se delineado um problema, a partir dos pontos-chave acima discutidos: Como avaliar se as informações sobre drogas disponibilizadas em sites brasileiros são confiáveis e dignas de credibilidade?

\section{Aprofundando o tema: como reconhecer a qualidade da informação}

O problema configurava-se com dois temas específicos: drogas de abuso e internet. Os estudos encontrados não responderam especificamente às perguntas iniciais, mas serviram para nortear a elaboração de um trabalho capaz de ter aplicação na realidade.

O processo de pesquisa de avaliação de sites (Portal, 2009) contou com uma estrutura de apoio que abrangia recursos de informática (computadores, impressoras, acesso à internet e seus serviços como, por exemplo, world wide web (www), correio eletrônico), recursos bibliográficos (livros e periódicos científicos) e recursos humanos. Neste caso, tinha-se a supervisão de duas especialistas no tema drogas de abuso e dependência química, que facilitavam a resolução de problemas quanto a esses aspectos, além da orientação de uma especialista em informática. Desse modo caracterizava-se o processo multidisciplinar, formando-se, então, a rede de amparo para o conhecimento.

Inicialmente foram pesquisados estudos que abrangessem as questões levantadas para serem aplicados à nossa realidade, objetivando-se encontrar respostas para o problema destacado: 'Como avaliar se as informações sobre drogas disponíveis em sites brasileiros são corretas e confiáveis.'

$\mathrm{Na}$ ausência de uma escala brasileira que contemplasse tais necessidades de confiabilidade, optou-se por estudar trabalhos que abordassem a questão de avaliação de sites. Após a análise de algumas referências (Gunther, 
2002b; Lopes, 2004; Risk, 2005; Walji, 2004; Hiti, 1999), concluiu-se que seria possível criar um protocolo específico, voltado para a nossa demanda.

Nesse processo, os especialistas preocupavam-se com a aprendizagem decorrente da realização do trabalho que estava sendo conduzido pelos estudantes. Assim, esses profissionais tinham o papel de facilitar a aprendizagem e orientar a busca de resoluções para os problemas encontrados, estimulando a descoberta e utilização de diferentes recursos.

A primeira hipótese foi recorrer aos orientadores, com a ideia de que se teria uma solução imediata para o problema de como avaliar a credibilidade das informações sobre drogas que são disponibilizadas em sites brasileiros. Entretanto, ocorreram mais questionamentos do que respostas prontas. O intuito era de incentivar a busca por justificativas que subsidiassem a afirmação de que determinados sites, contendo informações sobre drogas de abuso, pudessem ser considerados de boa qualidade ou não.

A segunda hipótese foi recorrer a escalas existentes para a demanda em questão, com vistas a encontrar um estudo que solucionasse o problema de pesquisa. Porém, não se obteve uma única resposta como, por exemplo, uma escala ou um check list que pudesse ser utilizado ou traduzido para realizar a avaliação da qualidade da informação sobre drogas de abuso em sites brasileiros.

\section{Em busca de soluções: aprendendo com a realidade}

Considerando que, muitas vezes, os conteúdos disponibilizados na internet visam a atingir um público leigo, algumas entidades abordam questões de padrões de conduta para sites de saúde na internet, apresentando orientações para criação ou identificação de sites com qualidade e credibilidade em informação de saúde. Com base nas orientações estabelecidas por organizações reconhecidas internacionalmente, tais como: Health on the Net (HON), Health Information Technology Institute (Hiti) e American Medical Association (AMA) (Hiti, 1997; HONCode, 2008; Winker et al., 2000), iniciou-se a construção de um protocolo específico para avaliação de sites brasileiros que oferecem informações sobre drogas de abuso.

Durante a fase de criação do protocolo específico, cada critério descrito pela HON, Hiti e AMA foi questionado entre os acadêmicos. Discutia-se se o critério deveria ou não fazer parte do protocolo, justificando-se a decisão acordada. Para não haver precipitação durante esse processo, todas as ações eram intercaladas por reflexões, que avaliavam o método, ponderavam sobre os questionamentos e contextualizavam cada problema: se o caminho seria viável de ser seguido e se a sua implantação era necessária.

Quando as discussões envolviam questões de conteúdo, procurou-se delimitar os tópicos em função de características que são consideradas importantes 
ao tema. Definiu-se que os critérios de avaliação deveriam abranger os tópicos: ações e interações farmacodinâmicas e farmacocinéticas das substâncias, formas de uso, sinais e sintomas, efeitos psicológicos e sistêmicos do uso agudo e crônico, dados epidemiológicos, questões de diagnóstico, fatores de risco e proteção associados ao uso, prevenção, tratamento e políticas públicas.

A ferramenta de busca escolhida para realizar a pesquisa dos sites foi o Google (http://www.google.com.br), uma vez que é um dos principais mecanismos de busca nacional e internacional (Motta e Motta, 2002). Na sequência, definiram-se as palavras que seriam utilizadas na pesquisa (ou a expressão de busca) e, também, critérios para seleção das páginas que seriam avaliadas. Assim, para realizar um estudo piloto, optou-se por trabalhar com uma única droga, a cocaína. Das páginas resultantes da pesquisa, estudar-se-iam somente aquelas de domínio brasileiro, excluindo-se as páginas que contivessem notícias no seu conteúdo ou as que fossem artigos científicos.

Então, com base no contexto vivenciado e no tema proposto, que envolveu a definição e a criação do protocolo, o estudo aprofundado do tema proposto (drogas de abuso), o estabelecimento da metodologia dos critérios de inclusão/exclusão de sites, partiu-se para aplicação à realidade.

\section{De volta à realidade: aplicação de nosso próprio instrumento}

O estudo piloto realizado avaliou sites que ofereciam especificamente informações sobre a droga cocaína (Santos et al., 2006; Portal, 2009). Toda a informação presente no site e que fosse referente ao conteúdo era verificada. Dados inerentes ao tema deveriam ser sempre referenciados e não poderiam estar incompletos. Essa avaliação de conteúdo foi fundamentada no livro-texto Goodman \& Gilman's The Pharmacological Basis of Therapeutics (Brunton e Lazo, 2006). Eventualmente, fazia-se necessário recorrer a outros tipos de recursos, como artigos que apresentavam o tema drogas de abuso - nesse caso, recorria-se a bases de dados biomédicas para proceder à pesquisa de material.

A aplicação do protocolo criado incluiu 150 sites que se enquadravam nos critérios de avaliação. O instrumento desenvolvido para avaliar informações sobre drogas de abuso na internet mostrava-se, inicialmente, eficiente para apontar aqueles que contemplassem, simultaneamente, bom conteúdo, boa acessibilidade e credibilidade. Entretanto, sentiu-se a necessidade de refinar o instrumento, bem como de rever os critérios utilizados para selecionar os sites que seriam avaliados.

Alguns itens do protocolo foram revistos, pois se constatou que não se aplicavam à proposta do estudo como, por exemplo, a presença ou não da informação do caráter complementar do conteúdo. Nesse caso, esse item foi excluído, pois a orientação da importância da consulta médica para evitar o 
autodiagnóstico e automedicação não se presta à utilização de drogas de abuso. Ocorreu também modificação com relação a critérios de inclusão/exclusão, que serviam para indicar quais páginas da internet seriam avaliadas pelo protocolo. Por exemplo: inicialmente não eram selecionados sites que apresentassem publicidade (critério de exclusão). Entretanto, observou-se que nem sempre a propaganda estava relacionada com a informação ou proposta do site. Optou-se, então, por dividir este critério em duas categorias: propaganda de produtos e serviços relacionados ao assunto buscado e propaganda de produtos e serviços não relacionados com os termos buscados. Todos os critérios e itens do protocolo foram criteriosamente examinados e os sites novamente avaliados. Os resultados foram validados pelas especialistas em drogas de abuso, as quais acompanhavam a evolução do trabalho.

\section{Um processo de aprendizagem significativo: resultados}

A partir da problematização de um contexto real, desenvolveu-se um protocolo de avaliação de sites que permitiu delinear um panorama sobre a informação on line acerca de drogas de abuso. Verificou-se que há um número expressivo de páginas da internet que contém informações provenientes da crença popular, oferecendo pouco suporte científico aos usuários.

Este foi um estudo gerador de hipóteses, desenhando um primeiro panorama do perfil das informações sobre drogas de abuso disponíveis na internet brasileira. Possibilitou a adaptação e teste dos critérios de avaliação obtidos da literatura internacional para avaliação do tema e cumpriu a função de nortear a etapa analítica subsequente, com a finalidade de levar ao usuário final respaldo científico para a seleção de informação adequada sobre drogas de abuso na internet.

Essa experiência desencadeou o estabelecimento de metas, cultivando o cumprimento de responsabilidades. Além disso, proporcionou-se o desenvolvimento de habilidades que permitem identificar recursos de autoaprendizagem. Ao longo desse processo, constatou-se que os espaços reservados para aprendizagem, durante a formação universitária, também estão garantidos para aqueles acadêmicos que integram os programas de iniciação científica. No ambiente de estudo e de trabalho, aprendeu-se a receber e a assimilar críticas. As habilidades de comunicação foram aprimoradas, uma vez que os diferentes pontos de vista deveriam ser fundamentados e respeitados.

\section{Discussão e conclusões}

Esse processo de aprendizagem, vivenciado por estudantes de graduação, durante o desenvolvimento da avaliação de sites brasileiros sobre drogas de 
abuso, assemelha-se à metodologia enfocada na Teoria da Problematização, de Neusi Aparecida Navas Berbel (1996), com o caminho metodológico do Arco de Maguerez, que é dividido em cinco etapas, como segue: Observação da realidade; Pontos-chave; Teorização; Hipóteses de solução; e Aplicação à realidade (Bordenave e Pereira, 2005).

Ficou evidente, mediante a observação da realidade, que é difícil encontrar, na internet, sites que apresentem informações seguras e confiáveis sobre drogas de abuso. Antes da realização deste trabalho, não se sabia justificar quais sites poderiam compor a 'lista de favoritos'. Entretanto, após a caracterização do problema e da teorização, foi possível levantar hipóteses. Em função de não se avistar um único método que pudesse ser aplicado para o tema em questão, foi criado e testado um protocolo para auxiliar o processo de seleção de sites brasileiros que abordam o tema drogas de abuso.

A partir deste trabalho, desenvolveu-se a habilidade de analisar e criticar um site, justificando a pertinência ou não de sua inclusão em uma lista de favoritos. É reconhecida a importância de se ter discernimento para estabelecer uma avaliação crítica da literatura científica com a realidade, possibilitando, assim, uma prática baseada em evidências (Sacket et al., 2003). A experiência mostrou-se motivadora, uma vez que viabilizou o progresso individual, pois da condição de passividade passou-se para a condição de agente transformador da própria realidade.

\section{Agradecimentos}

Os autores agradecem a Helena M. T. Barros, Cleidilene Ramos Magalhães, Paulo Roberto de Souza Ramos e Roberta Coutinho Carvalho. 


\section{Notas}

1 Terapeuta ocupacional. Consultor do Serviço Nacional de Orientações e Informações sobre a Prevenção ao Uso Indevido de Drogas (VivaVoz). Mestrando do Programa Phoenix EM Dynamics of Health and Welfare pela École des Hautes Études en Sciences Sociale, França.<va9ner@gmail.com>

Correspondência: Rua Sarmento Leite, 245, CEP 90050-170, 3. ${ }^{\circ}$ andar, VivaVoz, Porto Alegre, Rio Grande do Sul, Brasil.

2 Consultor VivaVoz. Acadêmica de medicina da Universidade Federal de Ciências da Saúde de Porto Alegre (UFCSPA), Porto Alegre, Rio Grande do Sul, Brasil. $<$ marcelleportal@gmail.com>.

3 Farmacêutica da Universidade Federal de Ciências da Saúde de Porto Alegre (UFCSPA), Porto Alegre, Rio Grande do Sul, Brasil. Coordenadora VivaVoz. Doutora em Ciências Médicas pela Universidade Federal de Ciências da Saúde de Porto Alegre (UFCSPA). $<$ mari@ufcspa.edu.br>

4 Professora adjunta de Farmacologia do Departamento de Ciências Básicas da Saúde da Universidade Federal de Ciências da Saúde de Porto Alegre (UFCSPA), Porto Alegre, Rio Grande do Sul, Brasil. Doutora em Fisiologia pela Universidade Estadual de Campinas (Unicamp).<ddantas@ufcspa.edu.br>

5 Professora titular de Farmacologia do Departamento Ciências Básicas da Saúde da Universidade Federal de Ciências da Saúde de Porto Alegre (UFCSPA), Porto Alegre, Rio Grande do Sul, Brasil. Coordenadora VivaVoz. Pós-Doutora em Neuropsicofarmacologia pela Tufts University.<helenbar@ufcspa.edu.br>

6 Professora assistente do Departamento de Educação e Informação em Saúde da Universidade Federal de Ciências da Saúde de Porto Alegre (UFCSPA), Porto Alegre, Rio Grande do Sul, Brasil. Mestre em Ciências da Computação pela Universidade Federal do Rio Grande do Sul (UFRGS). < carolina.st@terra.com.br>

\section{Referências}

ALHEIT, Peter; DAUSIEN, Bettina. Processo de formação e aprendizagens ao longo da vida. Educação e Pesquisa, São Paulo, v. 32, n. 1, jan./abr., 2006.

BARROS, Helena M.T. et al. Neuroscience education for health profession undergraduates in a call-center for drug abuse prevention. Drug and Alcohol Dependence, n. 98, p. $270-274,2008$.

BERBEL, Neusi A.N. Metodologia da problematização no ensino superior e sua con- tribuição para o plano da práxis. Semina, v. 17, n. esp., p. 7-17, 1996.

BORDENAVE, Juan Diaz; PEREIRA, Adair Martins. Estratégias de ensino-aprendizagem. Petrópolis: Vozes, 2005.

BRASIL. Publicação de contratos, editais, avisos e ineditorias. Processo n. ${ }^{\circ} 00187$. 002127/2004-64. Espécie: Termo de Convênio n. ${ }^{\circ}$ 015/2004. Diário Oficial da União (DOU). Brasília, DF, 17 dez. 2004. Seção 3, p. 212 . 
BRUNTON, Laurance L.; LAZO, John S. Goodman \& Gilman's The Pharmacological Basis of Therapeutics. New York: McGrawHill, 2006.

CANDEIAS, Nelly Martins Ferreira. Conceitos de educação e de promoção em saúde: mudanças individuais e mudanças organizacionais. Revista Saúde Pública, São Paulo, v. 31, n. 2, Apr., 1997.

CARLINI, Elisaldo Araújo et al. II Levantamento domiciliar sobre uso de drogas psicotrópicas no Brasil: estudo envolvendo as 108 maiores cidades do país, 2005. Brasília: Secretaria Nacional Antidrogas; São Paulo: Centro Brasileiro de Informações sobre Drogas Psicotrópicas, Universidade Federal de São Paulo; 2007.

COMITÊ GESTOR DA INTERNET NO BRASIL. CGI.Br. Pesquisa sobre o uso das tecnologias da informação e da comunicação no Brasil - TIC Domicílios e TIC Empresas 2009. São Paulo, 2010. Disponível em: $<$ www.cetic.br>. Acesso em: setembro 2010.

FLEMING, M. Missy; MURRAY, Megan. Medical Education Model for the Prevention and Treatment of Alcohol User Disorders U.S. Department of Health and Human Services, Public Health Service, National Institute of Health, National Institute on Alcohol Abuse and Alcoholism, Rockville, MD. 1998.

Prevention and treatment of alcoholrelated problems: an international medical education model. Acad. Med., v. 71, n. 11, p. 1204-1210, 1996.

FREIRE, Paulo. Pedagogia do oprimido. São Paulo: Paz e Terra, 2000.

GLOWNIAK, Jerry V.; BUSHWAY, Marilyn K. Computer networks as a medical resource; accessing and using the Internet. Jama, n. 271, p. 1934-1939, 1994.

GUNTHER, Eysenbach. The epidemiology of (mis)information. The American Journal of Medicine, n. 113, p. 763-765, 2002a.
GUNTHER, Eysenbach et al. Empirical studies assessing the quality of health information for consumers on the world wide web: a systematic review. Jama, v. 287, n. 20, p. 2691-2700, 2002b.

HITI. Health Information Technology Institute. Agency for health care policy and research criteria for assessing the quality of health information on the internet. McLean, VA, 1999. <Disponível em: <www.hitiweb. mitretek.org/docs/criteria.html $>$. Acesso em: mar. 2007.

HON Code of Conduct (HONcode) for medical and health web sites Health on the Net Foundation. Health on the Net Foundation. Disponível em: <www.hon.ch/HONcode/ Conduct.html>. Acesso em: mar. 2008.

LOPES, Ilza Leite. Novos paradigmas para avaliação da qualidade da informação em saúde recuperada na web. Ciência da Informação, Brasília, v. 33, n. 1, p. 81-90, 2004.

MOTTA, Valter T.; MOTTA, Leonardo R. Informações biomédicas na Internet. Porto Alegre: Médica Missau, 2002.

PORTAL, Marcelle M. et al. Informações de saúde na internet: protocolo para avaliação de sites sobre drogas de abuso. Journal of Health Informatics, n. 1, v. 1, set. 2009.

RISK, Ahmad; DZENOWAGIS, Joan. Review of Internet Health Information Quality Initiatives. Journal of Medical Internet Research, v. 3, n. 4, p. e28, 2001.

SACKETT, David L. et al. Medicina baseada em evidências: prática e ensino. Porto Alegre: Artmed, 2003.

SANTOS Jaqueline Rodrigues et al. Implementação do Arco de Maguerez como alternativa metodológica para validação da teoria da problematização de Paulo Freire. In: REUNIÃO ANUAL DA SBPC, 58., jul. 2006, Florianópolis. Anais..., Florianópolis, 2006. Disponível em: <www.sbpcnet.org.br/livro/ 58ra/SENIOR/RESUMOS/resumo_2793.html>. Acesso em: nov. 2007. 
SANTOS, Vagner et al. Escala brasileira de avaliação de sites sobre drogas de abuso: estudo piloto. In: CONGRESSO BRASILEIRO DE EDUCAÇÃO MÉDICA, 44., 2006, Gramado. Revista Brasileira de Educação Médica. Rio de Janeiro: Associação Brasileira de Educação Médica, v. 1. p. 8, 2006.

SANZ-VALERO, Javier; CASTIEL, Luis D.; WANDEN-BERGHE, Carmen; JUAN QUILIS, Verónica. Internet y la búsqueda de información en salud pública: desde la relevancia hacia la 'revelancia'. Gac. Sanit, v. 20, n. 2, p. 159-160, 2006.

SOUZA, Marilise Fraga et al. VivaVoz: análise de um ano de atividades do Serviço Nacional de Orientações e Informações Sobre a Prevenção do Uso Indevido de Drogas. In: CONGRESSO DA ASSOCIAÇÃO BRASILEIRA DE ÁlCOOL E OUTRAS DROGAS, 18., set. 2006, Santos. Anais... Santos, 2006.
WALJI, Muhammad et al. Efficacy of quality criteria to identify potentially harmful information: a cross-sectional survey of complementary and alternative medicine web sites. Journal of Medical Internet Research, v. 6, n. 2, p. e21, 2004.

WINKER, A. Margaret et al. Guidelines for Medical and Health Information Sites on the Internet: Principles Governing AMA Web Sites. Jama, v. 283, n. 12, p. 1600-1606, 2000 .

Recebido em 13/05/2009

Aprovado em 22/06/2010 